\title{
NONLINEAR CONTROL TECHNIQUES FOR MICRO ELECTROSTATIC ACTUATORS IN THE PRESENCE OF PARASITICS AND PARAMETRIC UNCERTAINTIES
}

\author{
${ }^{1}$ Mohammad H. Salah, ${ }^{1}$ Ahmad M. Al-Jarrah, and ${ }^{2}$ Enver Tatlicioglu \\ ${ }^{1}$ Department of Mechatronics Engineering, Hashemite University, Zarqa 13115, Jordan \\ ${ }^{2}$ Department of Electrical and Electronics Engineering, Izmir Institute of Technology, Urla, Izmir, 35430, Turkey \\ msalah@hu.edu.jo, jarrah@hu.edu.jo, envertatlicioglu@iyte.edu.tr
}

\begin{abstract}
In this paper, nonlinear control techniques are developed to control parallel-plate micro electrostatic actuators in the presence of parasitics and parametric uncertainties. The movable plate of the micro actuator is actively controlled utilizing the measurements of internal charge and movable plate's displacement. A velocity observer is designed to estimate the velocity of the plate that is needed for the control algorithm since it is difficult to be measured practically. The proposed backstepping nonlinear control strategies are developed based on a Lyapunov-based analysis, which proves that the desired plate's displacement can be obtained accurately. The proposed nonlinear controllers are capable of controlling the movable plate beyond the pull-in limit in the presence of parametric uncertainties. Representative numerical simulations are presented to demonstrate the performance of the developed nonlinear control strategies in accurately tracking desired deflections of the movable plate within the entire capacitive gap. Finally, a comprehensive performance comparison is performed to examine the effectiveness of the control designs.
\end{abstract}

\section{KEY WORDS}

Nonlinear control, MEMS, electrostatic actuators.

\section{Introduction}

Parallel-plate type micro electrostatic actuators are the most common micro electro mechanical systems (MEMS) due to their rapid speed, high force, and low power consumption. Micro electrostatic actuators have a wide range of significant applications such as precision positioning, optical scanners, prob-based data storage, large vertical displacement, fatigue testing of low-stress thin films, and blood vessel manipulation.

Past research on micro electrostatic actuators has focused on modeling the nonlinear dynamics [2] and developing closed-loop control techniques to stabilize the device. The pull-in instability phenomenon is still a wellknown yet challenging problem that needs to be solved [3]. The main challenge is to extend the travel range of the parallel plate micro electrostatic actuator beyond the pull-in limit that is one third of its full capacitive gap. Seeger and Crary [11] presented a simple method to stabilize the micro actuator by adding a series capacitance. This work was extended in Seeger and Boser [9] with a switched-capacitor circuit to stabilize the device against voltage pull-in. Lu and Fedder [5] used a pre-filter in front of the feedback loop to shape the input command. It has been later proven that instability is avoided by a sufficient reduction in the drive voltage to achieve full gap operation [8] and that was also verified when Bermejo and Castaner [1] studied the potential use of a photovoltaic source as direct drive of the micro electrostatic actuator.

Many control strategies have been developed to solve the pull-in instability problem of micro electrostatic actuators. Seeger and Boser [10] presented a circuit that controls the amount of charge on a parallel-plate electrostatic actuator. They showed that charge control increased the stable ranges of motion but the maximum stable deflection is limited due to parasitic capacitance and tip-in. Miathripala et al. [6] examined control strategies for electrostatically actuated MEMS to eliminate the pull-in bifurcation and stabilized any desired operating points in the capacitive gap. They also showed that significant improvement in transient behavior in lightly damped MEMS requires dynamic estimation of electrode velocity. Owusu and Lewis [7] presented an effective nonlinear tracking control strategy for micro electrostatic actuators to increase the range of stable motion to the entire capacitive gap. The authors of [12] linearized the micro electrostatic actuator model at multiple operating points and then designed a robust PID control switching scheme for set-point regulation. Zhu et al. [14] presented two robust control laws for a parallelplate electrostatic microactuator in the presence of parasitics and parametric uncertainties. Their proposed controllers demonstrated satisfactory and robust performance in numerical simulations.

In this paper, nonlinear output feedback controllers are proposed for parallel-plate micro electrostatic actuators in the presence of parasitics and parametric uncertainties. The measurements of displacement and internal charge of the micro actuator are employed in the control algorithms to accurately track desired time-varying deflections of the movable plate. The estimate of the movable plate's velocity is utilized as well since it is difficult to be measured practically. A Lyapunov-based analysis is utilized to develop the nonlinear control strategy to 
operate the micro actuator beyond the pull-in instability. Representative numerical results are presented which demonstrates the performance and proof of concept of the active control approach.

\section{Micro Actuator Dynamics with Parasitics}

The dynamics of a typical micro electrostatic actuator can be modeled as a spring-mass-damper system as shown in Figure 1. A series resistor is connected to the device to optimize the speed-energy characteristics which has an enormous effect on both the switching speed and energy requirements. Figure 2 shows the equivalent circuit of the micro actuator with the series and parallel parasitic capacitances.

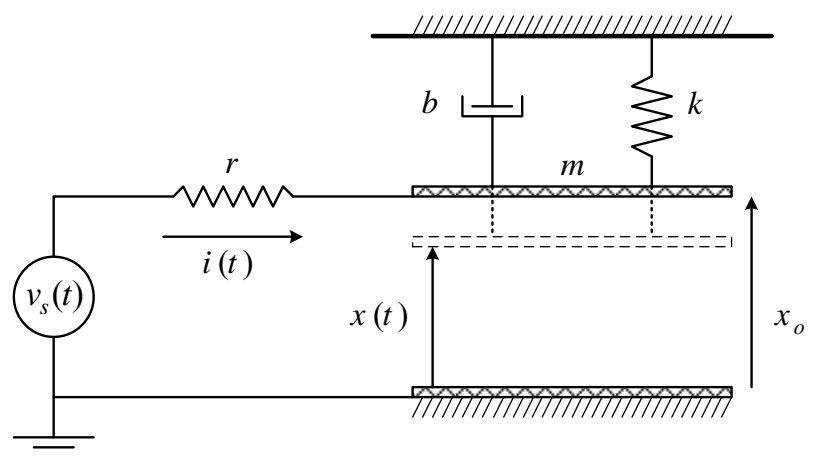

Figure 1. Micro electrostatic actuator model where only the top plate of the micro device is allowed to move.

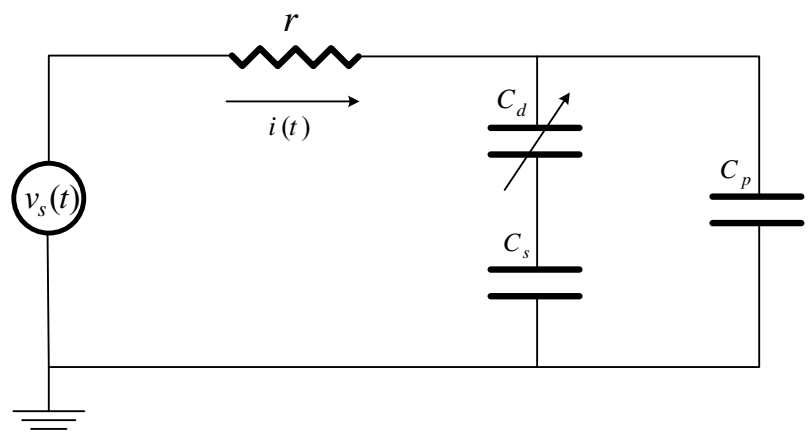

Figure 2. Equivalent circuit model with all parasitics and parametric uncertainties.

From Figures 1 and 2, the nonlinear dynamic model can be written as [14]

$$
\begin{gathered}
m \ddot{x}=-b \dot{x}-k\left(x-x_{o}\right)-\frac{q^{2}}{2 \varepsilon A} \\
\dot{q}=\frac{1}{r\left(1+\rho_{p} \rho_{s}+\rho_{p} \frac{x}{x_{o}}\right)}\left(v_{s}-\left(\frac{x}{\varepsilon A}+r \rho_{p} \frac{\dot{x}}{x_{o}}+\rho_{s} \frac{x_{o}}{\varepsilon A}\right) q\right)
\end{gathered}
$$

where $\quad x(t) \in \mathbb{R}^{+} \quad$ and $\quad \dot{x}(t), \ddot{x}(t) \in \mathbb{R} \quad$ are the displacement, velocity, and acceleration of the movable plate, respectively, $m \in \mathbb{R}^{+}$is the mass of the movable plate, $k \in \mathbb{R}^{+}$and $b \in \mathbb{R}^{+}$are the spring and damping constants, respectively, $x_{o} \in \mathbb{R}^{+}$denotes the maximum gap between the movable and fixed plates and also called the zero voltage gap, $q(t), \dot{q}(t) \in \mathbb{R}$ represent the charge and current of the micro actuator, respectively, $A \in \mathbb{R}^{+}$is the plate area, $\varepsilon \in \mathbb{R}^{+}$is the permittivity in the gap, $r \in \mathbb{R}^{+}$represents the series resistor, $v_{s}(t) \in \mathbb{R}$ denotes the voltage control input, and $\rho_{p}, \rho_{s} \in \mathbb{R}^{+}$denotes the parallel and series parasitic uncertainties. The parallel and series parasitic uncertainties are defined as $\rho_{p} \triangleq \frac{C_{p}}{C_{o}}$ and $\rho_{s} \triangleq \frac{C_{O}}{C_{s}}$ where $C_{p}, C_{s} \in \mathbb{R}^{+}$are the parallel and series parasitic capacitances, respectively, $C_{o} \in \mathbb{R}^{+}$is the capacitance of the micro device at rest and equal to $\frac{\varepsilon A}{x_{O}}$, and $C_{d} \in \mathbb{R}^{+}$denotes the capacitance of the micro device and equal to $\frac{\varepsilon A}{x}$.

Remark 1: The displacement of the micro actuator's movable plate, $x(t)$, is constrained with the condition $\delta_{o} \leq x(t) \leq x_{o}$ where $\delta_{o} \in \mathbb{R}^{+}$represents the thickness of the insulating material coated on the bottom plate. This material is mounted to prevent the micro actuator from getting a short-circuit condition. In addition, the existence of the series resistor, $r$, in the circuit is another protection from the occurrence of a short-circuit condition.

To facilitate the nonlinear control design, the micro actuator dynamic model described in (1) and (2) is normalized as

$$
\begin{gathered}
\ddot{X}=-2 \xi \dot{X}-X+1-\frac{Q^{2}}{3} \\
\dot{Q}=\beta\left[-Q\left(X+\rho_{s}\right)+R \rho_{p} \dot{X} Q+\frac{2}{3} V\right]
\end{gathered}
$$

where $X \triangleq \frac{x}{x_{o}}$ is the normalized displacement of the movable plate, $Q \triangleq \frac{q}{q_{p}}$ is the normalized micro actuator charge, $V \triangleq \frac{v_{s}}{v_{p}}$ is the normalized control voltage, $q_{p} \triangleq \frac{3}{2} C_{o} v_{p}$ is the pull-in charge, $v_{p} \triangleq \omega \sqrt{\frac{8 m x_{o}^{2}}{27 C_{o}}}$ is the pull-in control voltage, $C_{o} \triangleq \frac{\varepsilon A}{x_{o}}$ is the capacitance at rest, $\omega \triangleq \sqrt{\frac{k}{m}}$ is the undamped natural frequency, $\xi \triangleq \frac{b}{2 \sqrt{m k}}$ is the damping ratio, $R=r \omega C_{o}$ is a scaling constant, and $\beta \triangleq \frac{1}{\alpha}$ where $\alpha \triangleq R+R \rho_{p} \rho_{s}+R \rho_{p} X$. Note that $\dot{\alpha}=R \rho_{p} \dot{X}$. The normalized dynamics described in (3) and (4) are with respect to the normalized 
time $T$ which is equal to $\omega t$ where $t$ is the time in actual dynamics.

\section{Nonlinear Control Design}

The subsequent backstepping control development utilizes the fact that the normalized displacement, $X(T)$, and velocity, $\dot{X}(T)$, of the micro actuator's movable plate are measurable but since the velocity may not be available, a velocity observer will be designed in Section 4. The main control objective is to design the normalized voltage control input, $V(T)$, introduced in (4), to force $X(T)$ to track a desired plate displacement, denoted by $X_{d}(t) \in \mathbb{R}^{+}$, in the sense that

$$
X(T) \rightarrow X_{d}(t) \quad \text { as } \quad T \rightarrow \infty .
$$

The subsequent controller development requires that a desired time-varying trajectory is selected such that $X_{d}(T), \dot{X}_{d}(T)$, and $\ddot{X}_{d}(T)$ are bounded. It is also required that the device charge, $Q(T)$, tracks an auxiliary signal (desired charge), $Q_{d}(T) \in \mathbb{R}^{+}$, in the sense that

$$
Q(T) \rightarrow Q_{d}(T) \quad \text { as } \quad T \rightarrow \infty
$$

To facilitate the control design, the following error signals, $e(T) \in \mathbb{R}$ and $\eta(T) \in \mathbb{R}$, are defined

$$
\begin{aligned}
& e \triangleq X-X_{d} \\
& \eta \triangleq Q_{d}-Q .
\end{aligned}
$$

Based on the definitions of $e(T)$ and $\eta(T)$ in (7) and (8), respectively, it is clear that if $e(T), \eta(T) \rightarrow 0$ as $T \rightarrow \infty$, then $X(T) \rightarrow X_{d}(t)$ and $Q(T) \rightarrow Q_{d}(T)$ as $T \rightarrow \infty$; thus, meeting the control objectives in (5) and (6).

By taking the second time derivative of $e(T)$ in (7), the following expression may be obtained

$$
\ddot{e}=-2 \xi \dot{e}-e-\ddot{X}_{d}-2 \xi \dot{X}_{d}-X_{d}+1-\frac{Q^{2}}{3}
$$

where (3), (7) and its first time derivative were utilized. By adding and subtracting $\frac{Q_{d}^{2}}{3}$ to (9) and utilizing (7), the expression in (9) may be rewritten as

$$
\ddot{e}=-2 \xi \dot{e}-e-\ddot{X}_{d}-2 \xi \dot{X}_{d}-X_{d}+1-\frac{Q_{d}^{2}}{3}+\frac{\left(Q_{d}+Q\right)}{3} \eta
$$

where (8) was utilized.

To achieve the control objectives, the auxiliary signal, $Q_{d}(T)$, is designed as [13]

$$
Q_{d}=\sqrt{3\left(u_{F B}+\gamma\right)}
$$

where $u_{F B}(T) \in \mathbb{R}$ is the feedback control signal and $\gamma \in \mathbb{R}^{+}$is defined as

$$
\gamma \triangleq 1-X_{d}-2 \xi \dot{X}_{d}-\ddot{X}_{d} .
$$

Remark 2: $\quad$ In (12), $\quad \gamma(T)$ is bounded since its arguments are bounded as mentioned earlier and it can be proven that the constraint $u_{F B}>-\gamma$ is satisfied and it is to obtain a real value for the expression in (11).

After utilizing (11) and (12), the expression in (10) can be written as

$$
\ddot{e}=-2 \xi \dot{e}-e-u_{F B}+\frac{\left(Q_{d}+Q\right)}{3} \eta .
$$

The feedback control signal, $u_{F B}(T)$, is designed as

$$
u_{F B}=\frac{\gamma}{2}(\tanh (\lambda e)+\tanh (\lambda \dot{e}))
$$

where $\tanh (\cdot)$ is the hyperbolic tangent function and $\lambda \in \mathbb{R}^{+}$is a control gain. After utilizing (14), the error dynamics in (13) becomes

$$
\ddot{e}=-g(e)-h(\dot{e})+\frac{\left(Q_{d}+Q\right)}{3} \eta
$$

where the terms $g(e)$ and $h(\dot{e})$ are defined as

$$
\begin{gathered}
g(e) \triangleq e+\frac{\gamma}{2} \tanh (\lambda e) \\
h(\dot{e}) \triangleq 2 \xi \dot{e}+\frac{\gamma}{2} \tanh (\lambda \dot{e}) .
\end{gathered}
$$

Property 1: $\quad$ From the definitions in (16) and (17), it is clear that $g(0)=h(0)=0$ and $\tau g(\tau)>0$ as well as $\tau h(\tau)>0$ are satisfied for all $\tau$.

After taking the first time derivative of (8) and utilizing (4) and multiplying both sides of the resulting equation with $\alpha(X)$, the following error dynamics is obtained for $\eta(T)$

$$
\alpha \dot{\eta}=\alpha \dot{Q}_{d}+Q\left(X+\rho_{s}\right)-R \rho_{p} \dot{X} Q-\frac{2}{3} V .
$$

By adding and subtracting the term $\frac{1}{2} \dot{\alpha} \eta$ to the right hand side of (18), the following expression can be obtained

$$
\alpha \dot{\eta}=Y+Q X-\frac{1}{2} \dot{\alpha} \eta-\frac{2}{3} V
$$

where the function $Y(\cdot) \in \mathbb{R}$ is defined as

$$
Y \triangleq \alpha \dot{Q_{d}}+\left(\rho_{s}-\dot{\alpha}\right) Q+\frac{1}{2} \dot{\alpha} \eta
$$

where $\dot{Q}_{d}(t)$ is computed as

$$
\dot{Q}_{d}=\frac{3 \gamma \lambda}{4 Q_{d}}\left(\operatorname{sech}^{2}(\lambda e) \dot{e}+\operatorname{sech}^{2}(\lambda \dot{e}) \ddot{e}\right) .
$$

Remark 3: $\quad$ A velocity observer will be designed in Section 4 to facilitate the computation of the function $\dot{\alpha}(T)$ which is equal to $\dot{\alpha}=R \rho_{p} \dot{X}$.

In the subsequent sections, three controllers are proposed to compensate for parasitics and parametric uncertainties presented in the micro actuator dynamics while guaranteeing tracking of a desired deflection of the movable plate. 


\subsection{Exact Model Knowledge Controller}

To facilitate the exact model knowledge (EMK) controller design, it is assumed that the function $Y(\cdot)$, introduced in (19) and defined in (20), is known a priori (i.e., all system parameters are known). The voltage control input, $V(T)$, introduced in (19), is designed as

$$
V=\frac{3}{2}\left[Y+Q X+k_{s} \eta+\frac{1}{3}\left(Q_{d}+Q\right) \dot{e}\right]
$$

where $k_{s} \in \mathbb{R}^{+}$is a control gain, thus; the closed-loop error dynamics of $\eta(T)$ becomes

$$
\alpha \dot{\eta}=-\frac{1}{2} \dot{\alpha} \eta-k_{s} \eta-\frac{1}{3}\left(Q_{d}+Q\right) \dot{e} .
$$

By utilizing the Lyapunov function $V_{1}=\frac{1}{2} \dot{e}^{2}+\int_{0}^{e} g(\tau) \tau+\frac{1}{2} \alpha \eta^{2}$ and utilizing the controller, given in (11) and (22), it can be proven that $\dot{V_{1}} \leq-2 \xi \dot{e}^{2}-k_{s} \eta^{2}$ which implies that $e(T), \eta(T) \rightarrow 0$ as $T \rightarrow \infty$. Hence, $X(T) \rightarrow X_{d}(T)$ and $Q(T) \rightarrow Q_{d}(T)$ as $T \rightarrow \infty$ and all closed-loop signals are bounded. Note that complete Lyapunov analysis was omitted due to page restrictions.

\subsection{Adaptive Controller}

To facilitate the adaptive controller design, it is assumed that the function $Y(\cdot)$, introduced in (19) and defined in (20), is linearly parameterized as

$$
Y \triangleq W^{T} \theta
$$

where $W(t) \in \mathbb{R}^{5 \times 1}$ is the regression matrix and $\theta \in \mathbb{R}^{5 \times 1}$ is the vector of unknown parameters and they are defined as

$$
\begin{aligned}
& W \triangleq\left[\dot{Q}_{d}, \quad X \dot{Q}_{d}, \quad Q, \quad \dot{X} Q, \quad \dot{X} \eta\right]^{T} \\
& \theta \triangleq\left[\begin{array}{llll}
\left.R+R \rho_{p} \rho_{s}\right), & R \rho_{p}, \quad \rho_{s}, \quad R \rho_{p}, & \frac{1}{2} R \rho_{p}
\end{array}\right]^{T}
\end{aligned}
$$

The voltage control input, $V(T)$, introduced in (19) is designed as

$$
V=\frac{3}{2}\left[Q X+W^{T} \hat{\theta}+k_{s} \eta+\frac{1}{3}\left(Q_{d}+Q\right) \dot{e}\right]
$$

where $k_{s} \in \mathbb{R}^{+}$is a control gain and $\hat{\theta}$ is the estimate of the unknown system parameters and is computed from

$$
\dot{\hat{\theta}}=\Delta \eta W
$$

where $\Delta \in \mathbb{R}^{+}$is an adaptive update gain, thus; the closed-error dynamics of $\eta(T)$ becomes

$$
\alpha \dot{\eta}=W^{T} \tilde{\theta}-k_{s} \eta-\frac{1}{3}\left(Q_{d}+Q\right) \dot{e}
$$

where $\tilde{\theta} \in \mathbb{R}^{5 \times 1}$ is the estimation error and defined as

$$
\tilde{\theta} \triangleq \theta-\hat{\theta} \text {. }
$$

By utilizing the Lyapunov function

$V_{1}=\frac{1}{2} \dot{e}^{2}+\int_{0}^{e} g(\tau) \tau+\frac{1}{2} \alpha \eta^{2}+\frac{1}{2 \delta} \tilde{\theta}^{T} \tilde{\theta}$ and utilizing the controller, given in (11) and (27), it can be proven that $\dot{V_{1}} \leq-2 \xi \dot{e}^{2}-k_{s} \eta^{2}$ which implies that $e(T), \eta(T) \rightarrow 0$ as $T \rightarrow \infty$. Hence, $X(T) \rightarrow X_{d}(T)$ and $Q(T) \rightarrow Q_{d}(T)$ as $T \rightarrow \infty$ and all closed-loop signals are bounded. Note that complete Lyapunov analysis was omitted due to page restrictions.

\subsection{Robust Controller}

To facilitate the robust controller design, it is assumed that the function $Y(\cdot)$, introduced in (19) and defined in (20), can be upper bounded in the sense that $Y(\cdot) \leq \rho(\cdot)$ where $\rho(\cdot) \in \mathbb{R}^{+}$is a positive non-decreasing function. Based on the subsequent analysis and Assumption 4, the voltage control input, $V(T)$, introduced in (19), is designed as

$$
V=\frac{3}{2}\left[Q X+\rho \operatorname{sgn}(\eta)+k_{s} \eta+\frac{1}{3}\left(Q_{d}+Q\right) \dot{e}\right]
$$

where $k_{s} \in \mathbb{R}^{+}$is a control gain, thus; the closed-error dynamics of $\eta(T)$ becomes

$$
\alpha \dot{\eta}=Y-\rho \operatorname{sgn}(\eta)-k_{s} \eta-\frac{1}{3}\left(Q_{d}+Q\right) \dot{e} .
$$

By utilizing the Lyapunov function $V_{1}=\frac{1}{2} \dot{e}^{2}+\int_{0}^{e} g(\tau) \tau+\frac{1}{2} \alpha \eta^{2}$ and utilizing the controller, given in (11) and (31), it can be proven that $\dot{V_{1}} \leq-2 \xi \dot{e}^{2}-k_{s} \eta^{2}$ which implies that $e(T), \eta(T) \rightarrow 0$ as $T \rightarrow \infty$. Hence, $X(T) \rightarrow X_{d}(T)$ and $Q(T) \rightarrow Q_{d}(T)$ as $T \rightarrow \infty$ and all closed-loop signals are bounded. Note that complete Lyapunov analysis was omitted due to page restrictions.

Remark 4: $\quad$ The control laws designed in (22), (27), and (31) requires that the micro actuator's normalized charge $Q(T)$ is measurable. In fact, the measurement of the device actual charge $q(t)$ can be obtained by measuring the voltage drop, $v_{r}(t)$, across the series resistor, $r$, and actual micro actuator's movable plate displacement $x(t)$ so that $q=\frac{\varepsilon A}{x}\left(v_{s}-v_{r}\right)$ where the voltage drop across the device is $v_{\text {device }}=v_{s}-v_{r}=\frac{q x}{\varepsilon A}$. Recall that $Q=\frac{q}{q_{p}}$.

\section{Velocity Observer}

The velocity of the micro actuator's movable plate can not be directly measured during the normal operation of the device. Thus, a velocity observer is needed to estimate the 
velocity of the movable plate in order to implement the proposed control scheme. A reduced-order velocity observer can be designed [14]. The following signal is defined

$$
y \triangleq \dot{X}-k_{v} X
$$

where $k_{v} \in \mathbb{R}^{+}$is the observer gain. After taking the first time derivative of (33), the following expression can be obtained

$$
\dot{y}=-2 \xi \dot{X}-X+1-\frac{Q^{2}}{3}-k_{v} \dot{X}
$$

where (3) was utilized. The expression in (34) can be rewritten as

$$
\dot{y}=-\left(2 \xi+k_{v}\right) y-\left[\left(2 \xi+k_{v}\right) k_{v}+1\right] X+1-\frac{Q^{2}}{3}
$$

where (33) was utilized. After utilizing the expression in (35), it is possible to compute the estimate of $y(T)$ such that

$$
\dot{\hat{y}}=-\left(2 \xi+k_{v}\right) \hat{y}-\left[\left(2 \xi+k_{v}\right) k_{v}+1\right] X+1-\frac{Q^{2}}{3} \text {. }
$$

Hence, after utilizing (33), the estimate of the velocity can be implemented as

$$
\hat{\dot{X}}=\hat{y}+k_{v} X \text {. }
$$

To analyze the stability of the observer the following observer error is defined

$$
\tilde{y} \triangleq \hat{y}-y=\dot{\dot{X}}-\dot{X}
$$

where (33) and (37) were utilized. By taking the first time derivative of (38), the following expression can be obtained

$$
\dot{\tilde{y}}=-\left(2 \xi+k_{v}\right) \tilde{y}
$$

where (35) and (36) were utilized. From (39), it is clear that the observer error is globally exponentially stable at the origin.

Remark 5: $\quad$ The control laws presented in (14), (22), (27), and (31) require the signal $\dot{e}(T)$ to be known and that requires the velocity of the micro actuator's movable plate to be measurable in turn. The signal $\dot{X}(T)$ can be estimated from the observer design presented in (37), and since the signal $\hat{y}(T)$ is computable from (36), then $\dot{e}(T)$ can be computed.

\section{Numerical Simulation}

In this section, numerical simulations are presented to demonstrate the performance of the controllers introduced in (11), (14), (22), (27), and (31) for two cases. In Case I a constant desired set-point was applied, while in Case II a time-varying desired trajectory was applied. The system model parameter values were chosen such that $\mathrm{M}=3.29 \times 10^{-10}[\mathrm{~kg}], \quad \mathrm{K}=476[\mathrm{~N} / \mathrm{m}], \quad \mathrm{b}=8 \times 10^{-4}[\mathrm{~N} . \mathrm{s} / \mathrm{m}]$, $\varepsilon=8.85 \times 10^{-12}[\mathrm{~F} / \mathrm{m}], \mathrm{A}=6.2 \times 10^{-8}\left[\mathrm{~m}^{2}\right], \mathrm{r}=0.91 \times 10^{6}[\Omega]$, $x_{o}=2 \times 10^{-6}[\mathrm{~m}], \rho_{s}=1, \rho_{p}=0.5$, and X $(0)=0.05([4,14])$.
The control gains $\lambda, k_{s}, \delta$, and $\rho$ were tuned and selected to be $250,15,10$, and 7 , respectively, so that the best performance was achieved.

In Figure 3, the response of the micro actuator's actual normalized deflection is introduced for a desired set-point of $X_{d}=0.7$ showing the performance of all the designed controllers. The proposed nonlinear controllers were tested against measurement errors and a time shift. Independent zero-mean uniformly distributed noises with maximum amplitude equal to $15 \%$ of the set-point value was applied to the measurement of micro actuator displacement with a time shift of $\mathrm{T}=0.1$.

To further investigate the performance of the proposed controllers, a time-varying desired trajectory was applied, $X_{d}=0.4 \sin (0.5 \pi t)+0.45$, with the same parameter values listed in Table 1 and the same control gains. In addition, the proposed nonlinear controllers were tested against measurement errors and a time shift. Independent zero-mean uniformly distributed noises with a noise power equal to 0.00001 was applied with a time shift of $\mathrm{T}=0.1$. Figure 4 shows the normalized deflection of the micro actuator for the selected time-varying desired trajectory.

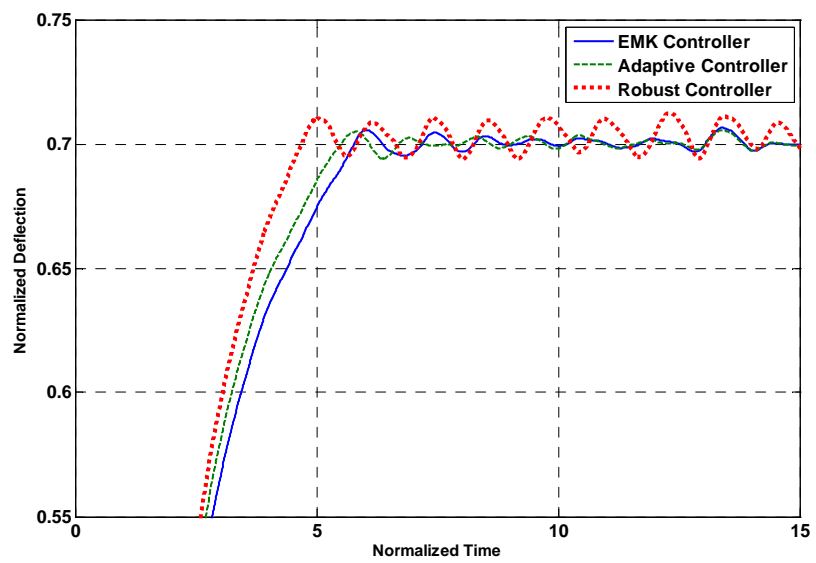

Figure 3. Normalized actual deflection for a desired setpoint of $X_{d}=0.7$ with measurement noise and time shift (Case I).

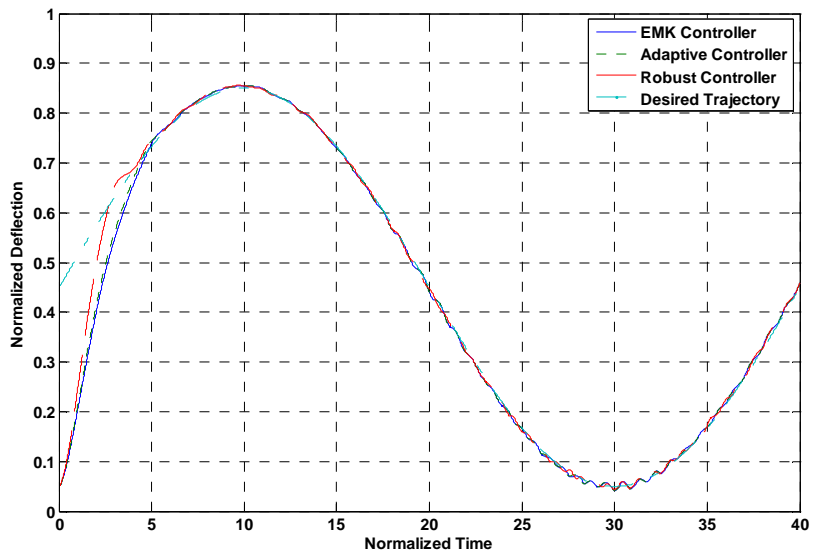

Figure 4. Normalized desired and actual deflection with measurement noise and time shift (Case II). 
In addition, the following measures were computed to quantify the performance of each controller,

$$
M_{e} \triangleq \int_{0}^{T}|e(\tau)|^{2} d \tau, \quad M_{u} \triangleq \int_{0}^{T}|V(\tau)|^{2} d \tau
$$

where $M_{e}(T)$ and $M_{u}(T)$ are measures of magnitude of the errors and the measure of energy expanded by the controller over the period of operation of the system $\mathrm{T}=15$ and $\mathrm{T}=40$ for Cases I and II, respectively. Table 1 shows a comparison of the performance for the proposed nonlinear controllers. It can clearly be seen from Table 1 that improved regulation and tracking performance is achieved by using the backstepping nonlinear robust output feedback controller. On the other hand, the effort of the robust controller, represented by $M_{u}(T)$, is a lot more than the other controllers with more shattering (voltage control inputs and internal charge figures were omitted due to page restrictions). However, the proposed controllers are capable of controlling the deflection of the micro actuator within the entire capacitive gap in the presence of the parasitics and uncertain parameters.

Table 1

Comparison of error and controller effort measures.

\begin{tabular}{|c||c|c||c|c|}
\hline \hline \multirow{2}{*}{ Controller } & \multicolumn{2}{c||}{ Case I } & \multicolumn{2}{c|}{ Case II } \\
\cline { 2 - 5 } & $M_{e}(T)$ & $M_{u}(T)$ & $M_{e}(T)$ & $M_{u}(T)$ \\
\hline \hline $\begin{array}{c}\text { Exact Model } \\
\text { Knowledge }\end{array}$ & 0.5803 & 352.4 & 0.2455 & 1803 \\
\hline Adaptive & 0.5613 & 349.9 & 0.2330 & 1863 \\
\hline Robust & 0.5098 & 1191 & 0.1861 & 5406 \\
\hline \hline
\end{tabular}

\section{Conclusion}

In this work, a backstepping nonlinear output feedback control strategy is developed for micro electrostatic actuators. The control algorithm is designed based on Lyapunov-type analysis where the measurements of the device's displacement and internal charge were utilized. A velocity observer was designed to facilitate the output feedback control technique. Numerical simulation results were presented to illustrate the performance of the nonlinear controller.

\section{References}

[1] Bermejo, S., and Castaner, L., "Dynamics of MEMS electrostatic driving using a photovoltaic source," Sensors and Actuators A: Physical, vol. 121, no. 1, pp. 237-242, 2005.

[2] Chu, P., Nelson, P., Tachiki, M., and Pister, K., "Dynamic of polysilicon parallel-plate electrostatic actuators," In Proc. of the 8th Int. Conf. on SolidState Sensors and Sctuators, and Eurosensors IX, Stockholm, Sweden, 1995, pp. 356-359.

[3] Fargas-Marques, A. and Casals-Terre, J., "Resonant pull-in condition in parallel-plate electrostatic actuators," IEEE Journal of Microelectromechanical Systems, vol. 16, no. 5, pp. 1044-1053, 2007.

[4] Goldsmith, C., Lin, T.-H., Powers, B., Wu, W.-R., and Norvel, B., "Micromechanical membrane switches for microwave applications," In Proc. of the IEEE MTT-S Int. Microwave Symposium Digest, Orlando, FL, 1995, pp. 91-94.

[5] Lu, M., and Fedder, G., "Closed-loop control of a parallel-plate microactuator beyond the pull-in limit," In Proc. of Solid-State Sensor, Actuator and Microsystems Workshop, Hilton Head Island, SC, 2002, pp. 255-258.

[6] Miathripala, D., Berg, J., and Dayawansa, W., "Control of an electrostatic microelectromechanical system using static and dynamic output feedback," ASME Journal of Dynamic Systems, Measurement, and Control, vol. 127, no. 3, pp. 443-450, 2005.

[7] Owusu, K., and Lewis, F., "Solving the "pull-in" instability problem of electrostatic microactuators using nonlinear control techniques," In Proceedings of the 2nd IEEE Int. Conf. on Nano/Micro Engineered and Molecular Systems, Bangkok, Thailand, 2007, pp. 1190-1195.

[8] Rocha, L., Cretu, E., and Wolffenbuttel, R., "Using dynamic voltage drive in a parallel-plate electrostatic actuator for full-gap travel range and positioning," IEEE Journal of Microelectromechanical Systems, vol. 15, no. 1, pp. 69-83, 2006.

[9] Seeger, J., and Boser, B., "Dynamics and control of parallel-plate actuators beyond the electrostatic instability," In Proc. of the 10th Int. Con. on SolidState Sensors and Actuators, Sendai, Japan, 1999, pp. 474-477.

[10] Seeger, J., and Boser, B., "Charge control of parallel-plate, electrostatic actuators and the tip-in instability," IEEE Journal of Microelectromechanical Systems, vol. 12, no. 5, pp. 656-671, 2003.

[11] Seeger, J., and Crary, S., "Stabilization of electrostatically actuated mechanical devices," In Proc. of the Int. Conf. on Solid-State Sensors and Actuators, Chicago, IL, 1997, pp. 1133-1136.

[12] Vagia, M., Nikolakopoulos, G., and Tzes, A., "Design of a robust PID-control switching scheme for an electrostatic micro-actuator," Control Engineering Practice, vol. 16, no. 11, pp. 13211328, 2008.

[13] Younis, M., Gao, F., and de Queiroz, M.S., "A Generalized Approach for the Control of MEM Relays," In Proc. of the American Control Conf., New York City, NY, 2007, pp. 3180-3185.

[14] Zhu, G., Penet, J., and Saydy, L., "Modeling and control of electrostatically actuated MEMS in the presence of parasitics and parametric uncertainties," ASME Journal of Dynamic Systems, Measurement, and Control, vol. 129, no. 6, pp. 786-794, 2007. 